\title{
Interrupción voluntaria del embarazo por violación: conocimientos, actitudes y prácticas de los profesionales de la salud en Caquetá
}

\author{
Voluntary interruption of pregnancy caused by rape: \\ a descriptive study on knowledge, attitudes \\ and practices of health professionals in Caquetá
}

Interrupção voluntária da gravidez por violação sexual: conhecimentos, atitudes e práticas dos profissionais de saúde em Caquetá

Marilyn Ibarguen S?

\section{RESUMEN}

El objetivo de este estudio descriptivo transversal fue describir los conocimientos, actitudes y prácticas de profesionales de la salud que laboran en el departamento de Caquetá sobre la interrupción voluntaria del embarazo a causa de violación en un contexto de conflicto armado. Para ello, se realizó una encuesta a 117 profesionales de la salud (médicos y enfermeras) de diferentes instituciones prestadoras de salud del departamento. Tamaño muestral calculado: 116, margen de error del $5 \%$ y nivel de confianza del $90 \%$. El $95 \%$ de los profesionales conoce que en Colombia es legal la interrupción voluntaria del embarazo cuando este es producto de una violación; el $17 \%$ manifestó que la denuncia es el único requisito para acceder a dicho procedimiento. El 50 \% respondió que la aspiración manual endouterina es uno de los métodos utilizados y el 62 \% consideró que la mujer embarazada a

Citación del artículo impreso:

Vancouver: Ibarguen M. Interrupción voluntaria del embarazo a causa de violación: conocimientos, actitudes y prácticas de los profesionales de la salud en Caquetá. Rev Colomb Enferm. 2017;14:56-64.

APA: Ibarguen, M. (2017). Interrupción voluntaria del embarazo a causa de violación: conocimientos, actitudes y prácticas de los profesionales de la salud en Caquetá. Revista Colombiana de Enfermería, 14, 56-64.

Citación del artículo en línea:

Vancouver: Ibarguen M. Interrupción voluntaria del embarazo a causa de violación: conocimientos, actitudes y prácticas de los profesionales de la salud en Caquetá. Rev Colomb Enferm [Internet]. 2017 [consultado (día mes año)];14. Disponible en: http://revistas.unbosque.edu.co/index.php/RCE o http://dx.doi.org/10.18270/rce.v14i.2028

APA: Ibarguen, M. (2017). Interrupción voluntaria del embarazo a causa de violación: conocimientos, actitudes y prácticas de los profesionales de la salud en Caquetá. Revista Colombiana de Enfermería, 14, 56-64. Recuperado de: http://revistas.unbosque.edu.co/index.php/RCE o http://dx.doi.org/10.18270/rce.v14i.2028

1. Enfermera, candidata a magíster en Salud Sexual y Reproductiva. Enfermera de Terreno, Organización Médicos del Mundo, Francia. Tumaco, Nariño, Colombia. Correo electrónico: marilynibarguensequeda@hotmail.com 
causa de violación debe hacer lo que considere correcto. El $47 \%$ de los encuestados dijo no practicar la interrupción voluntaria del embarazo (IVE) pero sí remitir el caso. Se identificó que los profesionales tienen información relacionada con la atención a víctimas de violencia sexual. Aun así, hay algunas dificultades en el reconocimiento formal de los requisitos para prestar este servicio ya que, en zonas de conflicto armado, las pacientes refieren que su integridad física podría verse comprometida si interponen una denuncia por violencia sexual, por lo cual la interrupción del embarazo se puede realizar bajo la causal salud. Además, se logró evidenciar que los profesionales prefieren remitir los casos de IVE.

Palabras clave: aborto legal; violación; conflicto armado; conocimientos, actitudes y prácticas en salud; responsabilidad legal.

\section{ABSTRACT}

Objective. To describe the knowledge, attitudes and practices of health personnel of the department of Caquetá in relation to voluntary termination of pregnancy because of rape. Material and methods. In this cross-sectional, descriptive study, a survey was carried out on knowledge, attitudes and practices in relation to the voluntary termination of pregnancy due to the rape in the context of an armed conflict. A total of 117 health professionals (doctors and nurses) of various health providing institutions in the department of Caquetá underwent a survey. Calculated sample size: 116, margin of error of $5 \%$ and confidence level of $90 \%$. Results. $95 \%$ of the professionals know that the voluntary termination of pregnancy is legal in Colombia when the pregnancy is caused by rape; filing a police report is the only requirement to access such procedure, $17 \%$ said; $50 \%$ responded that manual vacuum aspiration is a method used and $62 \%$ considered that the women impregnated by rape should do what she considers correct. Finally, $47 \%$ of those surveyed said they did not practice the voluntary termination of pregnancy (IVE for its), but would refer it. Conclusions. It was identified that professionals have information related to the care of victims of sexual violence. Even so, there are some difficulties in the formal recognition of the requirements to provide this service since, in areas of armed conflict, patients report that their physical integrity could be compromised if they file a report for sexual violence, a reason for the interruption to be done under the cause of health. In addition, it was possible to show that professionals prefer to refer IVE cases and not to practice them directly.

Key words: abortion, legal; rape; armed conflicts; health knowledge, attitudes, practice; liability, legal.

\section{RESUMO}

O objetivo deste estudo descritivo transversal foi descrever os conhecimentos, atitudes e práticas de profissionais de saúde que trabalham no departamento de Caquetá sobre a interrupção voluntária da gravidez devido à violação sexual em um contexto de conflito armado. Para isso, realizou-se uma pesquisa com 117 profissionais de saúde (médicos e enfermeiras) de diferentes instituições de saúde do departamento. Tamanho da amostra calculada: 116, margem de erro de 5\% e nível de confiança de 90\%. 95\% dos profissionais sabem que na Colômbia é aceita por lei a interrupção voluntária da gravidez quando é causada por violação sexual, 17\% disseram que a denúncia é o único requisito para ter acesso a tal procedimento. 50\% responderam que a aspiração manual a vácuo é um dos métodos utilizados e $62 \%$ consideraram que a mulher grávida devido à violação deve fazer o que considerar correto. 47\% dos entrevistados afirmaram não praticar a interrupção voluntária da gravidez (IVE), mas repassam o caso. Identificouse que os profissionais têm informações relacionadas ao atendimento às vítimas de violência sexual. Porém, ainda há algumas dificuldades no reconhecimento formal dos requisitos para prestar este serviço, uma vez que, em zonas de conflito armado, as pacientes explicam que sua integridade física pode ser comprometida caso prestem queixa. O aborto, portanto, pode ser realizado sob a causa saúde. Além disso, observou-se que os profissionais preferem passar para outros a responsabilidade sobre os casos de interrupção voluntária da gravidez (IVE).

Palavras-chave: aborto legal; estupro; conflito armado; conhecimentos, atitudes e prática em saúde; responsabilidade legal.

\section{INTRODUCCIÓN}

Según la Organización Mundial de la Salud (1), se calcula que cada año se producen 22 millones de abortos inseguros en el mundo, casi todos $(98$ $\%)$ en países en vías de desarrollo. Se entiende por aborto inseguro un procedimiento para finalizar un embarazo no deseado, realizado por personas que carecen de la capacitación necesaria o que se lleva a cabo en un entorno donde se carece de un estándar médico mínimo, o ambos (1). Entre el $10 \%$ y el 20 $\%$ de las mujeres que abortan necesitan atención 
médica debido a complicaciones, algunas como resultado de prácticas autoinfligidas o provocadas por personal con escasas habilidades para ello. Entre estas complicaciones figuran: aborto incompleto (no se retiran o se expulsan del útero todos los tejidos embrionarios), hemorragias (sangrado abundante), infección, perforación uterina (cuando se atraviesa el útero con un objeto afilado), y daños en el tracto genital y órganos internos debidos a la introducción de objetos peligrosos en la vagina o el ano (2). Aunque acudan a instituciones de salud, en algunos casos estas mujeres se estarían enfrentando a abortos inseguros, debido a que los servicios de atención postaborto en América Latina son generalmente de mala calidad por dificultades para el acceso, falta de oportunidad, uso de métodos inapropiados y actitudes prejuiciosas por parte del personal de las clínicas y los hospitales (3). Las más afectadas son las mujeres de bajos recursos en zonas rurales, que acceden a prácticas con personal no capacitado y por tanto corren mayor riesgo de complicaciones (4). En salud pública los gastos que se asumen en la atención de una complicación de un aborto inseguro se pueden reducir, y así beneficiar a más mujeres si se realizara un aborto seguro (5).

En algunos casos de embarazos por violación las mujeres no desean continuar con la gestación y recurren a diferentes medios para interrumpirla. Sin embargo, son muchas las barreras que se imponen a la garantía de los derechos sexuales y reproductivos, entre ellas: a) el desconocimiento por parte de la población de cuáles son sus derechos y deberes en materia de salud sexual y reproductiva (SSR); b) el desconocimiento o la negativa a realizar la interrupción del embarazo, algunos por ignorancia o prejuicio, otros por motivos de conciencia; c) la falta de entrenamiento del personal de salud para abordar la SSR con una perspectiva integral y de derechos; y d) la falta de claridad para orientar y aplicar los métodos modernos de anticoncepción y de interrupción voluntaria del embarazo (IVE), cuando sea indicada según los casos contemplados en la Sentencia C-355 de 2006 (6). El objetivo de realizar este estudio fue describir los conocimientos, actitudes y prácticas del personal de salud del departamento de Caquetá en relación con la IVE a causa de violación.

\section{Contexto de Caquetá}

El departamento de Caquetá se encuentra localizado al sur de Colombia, tiene 477.642 habitantes, está dividido en 16 municipios, limita al norte con los departamentos del Meta y Guaviare, al sur con Putumayo y Amazonas, al este con Vaupés y Amazonas. Caquetá presenta el ecosistema de Sabana Natural del Yarí, que sirve de refugio a gran cantidad de fauna y forma parte del gran corredor biológico andino-amazónico, y su principal renglón económico es la ganadería bovina (7).

Sus características geográficas son propicias para que los grupos irregulares se ubiquen en esa región, lo cual constituye un factor de riesgo para que se presenten casos de violencia sexual. Esta situación parece ser mucho más frecuente de lo que indican los datos reportados por las instituciones, puesto que, de acuerdo con la experiencia de la investigadora, las mujeres que han sido víctimas de violación en el marco del conflicto armado con frecuencia solicitan atención después de meses o años de ocurrido el hecho.

Según el Ministerio de la Protección Social, entre el 2007 y el 2009, se realizaron en el país un total de 623 procedimientos de interrupción voluntaria del embarazo, datos que fueron obtenidos con base en reportes de 16 secretarías de salud (8), sin incluir la Secretaría de Salud del Caquetá. Puede suponerse que dichos procedimientos no se desarrollaron por falta de demanda, no se reportaron casos a pesar de haberse realizado o simplemente no se solicitaron. Aunque al parecer a nivel nacional ha aumentado el acceso a la IVE en instituciones de salud desde que se emitió la Sentencia C-355 de 2006, el colectivo de organizaciones y personas "La Mesa para la Vida y la Salud de las Mujeres” publicó en 2011 un artículo en el cual menciona ciertos obstáculos para el acceso a este servicio, entre ellos, algunos relativos a los proveedores, como el desconocimiento de la sentencia, la falta de entrenamiento médico y la objeción de conciencia. Otros más son la falta de campañas educativas y la información parcial o equívoca que reciben las mujeres (9). Lo anterior lleva a preguntarse si esto mismo está ocurriendo en el departamento de Caquetá, con la particularidad de ser una zona de conflicto armado y el riesgo que esto representa para las mujeres. 


\section{MATERIALES Y MÉTODOS}

Este es un estudio prospectivo transversal, basado en una encuesta sobre conocimientos, actitudes y prácticas, realizada a enfermeros y médicos de diferentes instituciones prestadoras de servicios (IPS) en 7 de los 16 municipios del departamento de Caquetá, Colombia. Se consideraron como criterios de inclusión aquellos profesionales de la salud (enfermeros y médicos) que intervinieran en la ruta directa o indirecta de atención a víctimas de violencia sexual y formaran parte de los servicios de urgencias, hospitalización, consulta externa o administrativos.

Se realizó un cálculo de muestra con un universo de 200 profesionales, con un margen de error del $5 \%$ y un nivel de confianza del $90 \%$, lo que dio un total de muestra de 116 . Las encuestas se realizaron entre octubre del 2014 y junio del 2015. Se acudió a cada una de las IPS y se solicitó la participación de profesionales que cumplieran el criterio de inclusión. Se les explicó el objetivo de la encuesta a todos los interesados. A quienes accedieron se les entregó un documento físico.

La encuesta se diseñó con base en las utilizadas en otros estudios $(12,14,15)$ que abordaron el mismo tema. La encuesta consistió en la recolección de datos sociodemográficos y 15 preguntas cerradas divididas así: 5 sobre conocimientos, 5 sobre actitudes y 5 sobre prácticas del personal de salud respecto a la interrupción voluntaria del embarazo a causa de violación. Para verificar la validez de contenido, el instrumento se sometió al juicio de expertos captados individualmente, a partir de cuyas observaciones se hicieron los ajustes correspondientes. Participaron como expertos tanto médicos como enfermeros que trabajan en el área de ginecobstetricia, la mayoría de ellos profesores universitarios. Los datos recolectados se tabularon en Excel y luego se exportaron a SPSSS para el tratamiento estadístico. Como consideraciones éticas se tuvo en cuenta el artículo 11 de la Resolución 008430 de 1993 (10). Se concluyó que la investigación no tendría ningún riesgo, ya que no se identificaron ni trataron aspectos sensibles de la conducta. Sin embargo, por ser un tema relacionado con la ética y la moral, se informó a los participantes que podían retractarse si se sentían intimidados y no deseaban entregar el instrumento diligenciado. Las encuestas fueron anónimas y la investigadora garantizó la custodia de la información, de manera que ningún participante ni institución tuvo acceso a ellas, una vez diligenciadas, ni a los formatos de consentimiento informado.

\section{RESULTADOS}

Participaron 117 profesionales cuyas principales características fueron: el $45 \%$ laboraba en el servicio de urgencias, el $19 \%$ en consulta externa, el $12 \%$ en el área administrativa, el mismo porcentaje en servicios de ginecobstetricia, el $10 \%$ en el servicio de hospitalización y el $2 \%$ en cirugía.

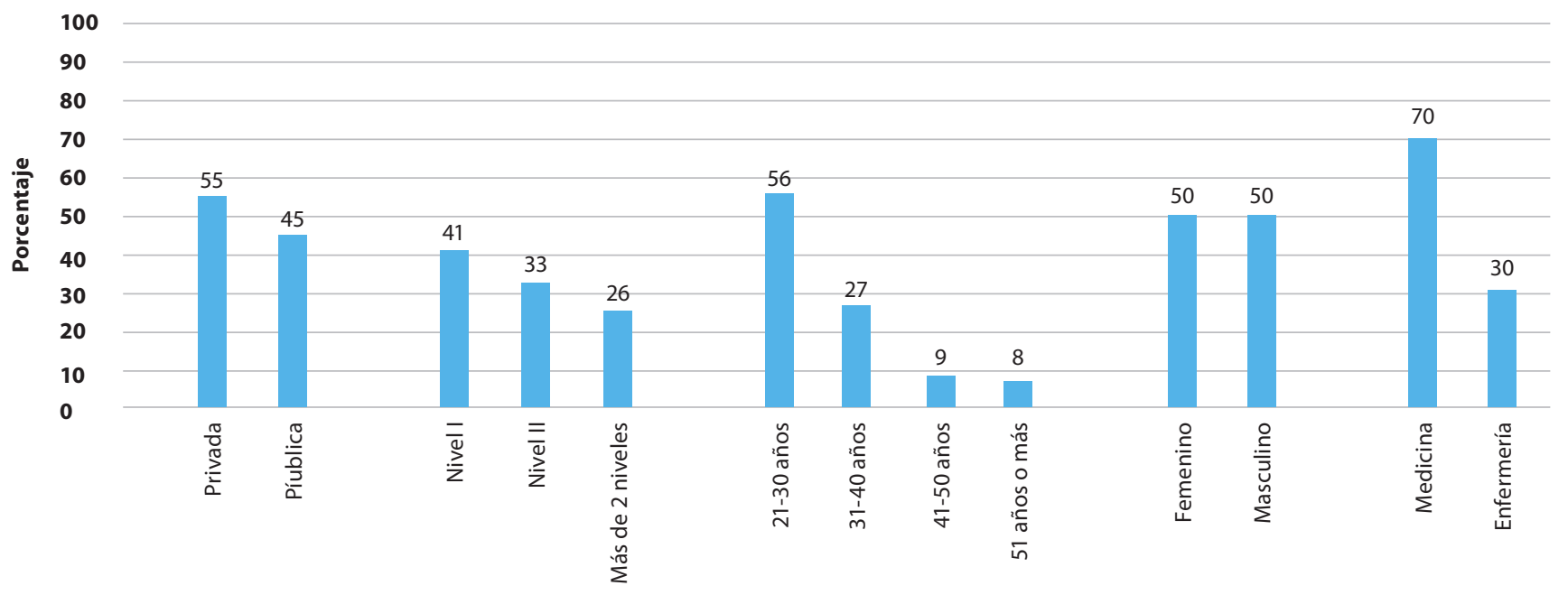

Figura 1. Descripción de participantes del estudio 
El $74 \%$ de los encuestados refirió haber recibido capacitación con relación a la atención de víctimas de violencia sexual, mientras el $26 \%$ manifiesta no haberla recibido. El $58 \%$ de los profesionales dijo haber sido capacitado en IVE mientras el $42 \%$ no lo había sido. El cuestionario no solicita información sobre los antecedentes de las capacitaciones, si fueron durante el tiempo académico, por iniciativa institucional laboral o por interés personal. El 15 $\%$ de los participantes manifestó haber realizado la declaración como objetor de conciencia ante la institución donde labora, mientras el $85 \%$ no lo había hecho.

En relación con los datos demográficos (véase Figura 1), los profesionales del primer nivel de atención predominaron con un $41 \%$, y el $55 \%$ de los encuestados laboraban en instituciones privadas. El rango de edad con mayor participación fue de 21-30 años, y los profesionales de medicina fueron quienes tuvieron mayor participación con un $70 \%$.

\section{Conocimientos}

A la primera pregunta, relacionada con el modelo de atención integral a víctimas de violencia sexual, el $82 \%$ de profesionales respondió que conoce de la existencia y aplicación del Protocolo y Modelo de Atención expedido en el 2011, el $9 \%$ no sabe acerca de ello, el $7 \%$, que está bajo modificaciones y el $2 \%$ señaló que no existe. En respuesta a la segunda pregunta, el $99 \%$ respondió que el embarazo forzado, la violación y los manoseos se catalogan como actos de violencia sexual. Para el 1 $\%$, el desplazamiento forzado, así como la atención y reparación a víctimas eran acciones de violencia sexual, y ningún participante refirió que no sabía o que el reclutamiento forzado con la anticoncepción definitiva formaban parte de este delito, aun cuando eran opciones que estaban contempladas. $\mathrm{Al}$ interrogante sobre los casos en los que la práctica del aborto es legal para la ley colombiana, el $95 \%$ de los profesionales encuestados respondió que el embarazo a causa de violencia sexual es uno de ellos, el $3 \%$, que en ninguna circunstancia es legal, un $1 \%$ no sabe y para otro $1 \%$ es legal cuando la pareja de la mujer lo solicita. En respuesta a los requisitos necesarios para practicar una IVE (véase Figura 2) el $50 \%$ de los encues- tados considera que se deben reunir los requisitos de la denuncia, el dictamen judicial, la certificación médica por parte del ginecólogo y el dictamen de la junta médica para acceder a la interrupción del embarazo.

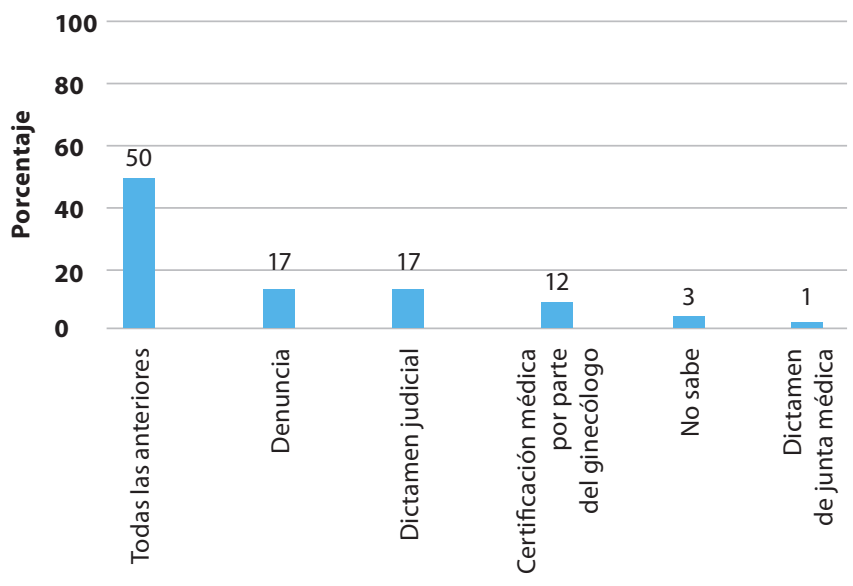

Figura 2. Conocimiento sobre los requisitos utilizados para acceder a una IVE

Los resultados sobre el conocimiento de los métodos para la realización de una IVE muestran que el $44 \%$ de los participantes desconoce la aspiración manual endouterina (véase Figura 3).

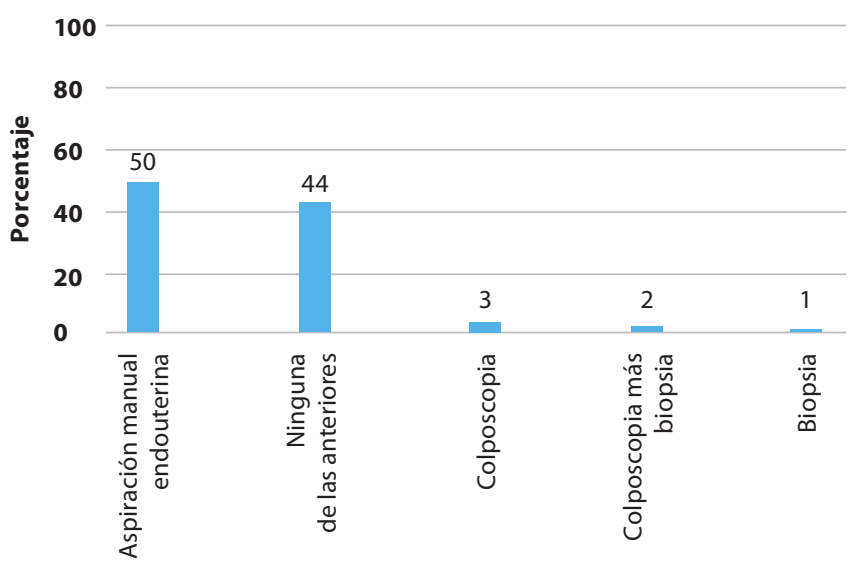

Figura 3. Conocimiento sobre el método utilizado para realizar una IVE

\section{Actitudes}

En cuanto a la opinión acerca de lo que debe hacer una mujer que haya quedado embarazada a causa de violación, el $62 \%$ de los encuestados dijo que debe hacer lo que considere correcto al respecto (véase Figura 4). 
El $79 \%$ de los encuestados expresó que una persona que ejerza la prostitución y haya sido víctima de violencia sexual debe recibir atención médica y psicológica; para el $6 \%$, el trabajo justifica la acción de violencia, para el $5 \%$ la situación fue provocada, y el $3 \%$ expuso que era una consecuencia del consumo de droga. El 7 \% respondió a la pregunta "todas las anteriores", es decir, cree que las drogas, el trabajo y alguna situación provocada son factores que llevan a que ocurra una violación en una persona que se prostituye; sin embargo, reconoce que debe recibir atención médica y psicológica.



Figura 4. Actitud sobre lo que debe hacer una mujer que ha quedado embarazada por violación

Con relación a la opinión sobre lo que debe pasar con una mujer que solicita la IVE a causa de violación, el $74 \%$ de los encuestados planteó que esta debe recibir orientación, el $23 \%$ respondió que debe atenderse su solicitud de manera inmediata. El $2 \%$ consideró que debe ser operada para no tener más hijos y el $1 \%$ que debe ir a la cárcel.

El $58 \%$ de los encuestados opinó que si la ley se hace más permisiva frente al aborto su práctica aumentará, un $25 \%$ respondió que seguirá igual, $14 \%$ manifestó no saber qué sucederá y para un $3 \%$, disminuirá.

Un $23 \%$ de los profesionales manifestó que la práctica de la IVE debería ser legal cuando corre peligro la vida de la mujer, cuando el embarazo ha sido producto de violación y cuando el feto presenta malformaciones, en el mismo porcentaje. Para el $11 \%$, esta debe hacerse cuando corre peligro la salud de la mujer, el mismo porcentaje cuando ha sido a causa de inseminación artificial no consentida; en caso de dificultades socioeconómicas y cuando la mujer toma la decisión, 2 \%. Un $1 \%$ respondió que debía practicarse la IVE en los siguientes casos: si el anticonceptivo falló, cuando la mujer es soltera, todas las razones son válidas para legalizar la práctica y en ningún caso debería ser legal.

\section{Prácticas}

En la práctica médica, el $92 \%$ de los encuestados respondió que cuando una mujer que ha sido víctima de violación sexual hace menos de 72 horas acude a consulta, primero debe recibir atención y luego orientación para hacer la denuncia, el $6 \%$ le suministraría tratamiento de acuerdo a la sintomatología y el $2 \%$ refirió que primero debe denunciar y luego recibir la atención médica.

Con relación a la asistencia al control prenatal de una menor de 14 años, el $38 \%$ de los profesionales respondió que prioriza la atención por parte de la institución que protege al menor, el 34 \% , que realiza valoración clínica de calidad y vida familiar, el $14 \%$ manifestó que indaga sobre el padre del hijo, el 13 $\%$, que la ingresa o continúa con el control prenatal normal, y el 1 \% que no sabía qué hacer.

El $47 \%$ de los profesionales señaló que si le solicitaran practicar una IVE no la realizaría, pero referiría a la paciente (véase Figura 5).

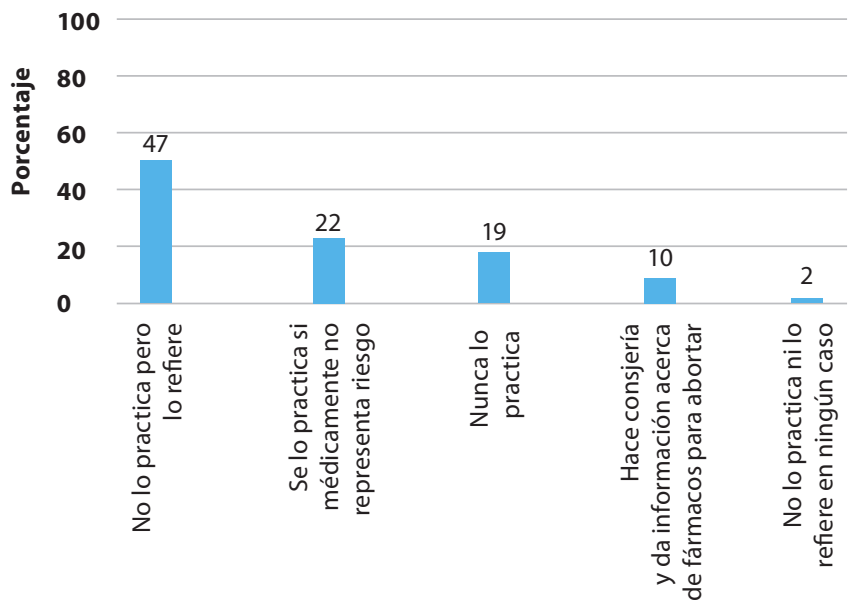

Figura 5. Procedimiento profesional por realizar cuando le solicitan una IVE 
El $21 \%$ que manifestó que nunca lo practicaría ni lo referiría justificó su respuesta así: 34 \% por razones éticas y morales, $31 \%$ por temor a estigma profesional, $16 \%$ por razones religiosas, el mismo porcentaje refirió otras razones, y $3 \%$ de los encuestados respondió que lo haría por el riesgo de complicaciones médicas.

Con relación al trato que recibe una paciente cuando llega con complicaciones de aborto a la institución donde trabaja, el $99 \%$ manifestó que se le respetan sus derechos, mientras el $1 \%$ señaló que no. En cuanto al apoyo psicológico, para el 85 \% sí se le brinda, pero para el $15 \%$, no. El $91 \%$ señaló que recibe atención médico-quirúrgica, el $9 \%$, que no. Con relación a la asesoría en anticoncepción antes de enviarla a casa, el $82 \%$ respondió que sí se le brinda, mientras el $18 \%$ refirió que no.

\section{DISCUSIÓN}

La violencia sexual como arma de guerra es una práctica de varias décadas, que tiene gran impacto en ocasionar terror colectivo e individual en contexto de violencia armada y política (11). Las víctimas de este delito tienen necesidades que en algunos casos no son suplidas; una de ellas es velar por su seguridad y prevenir nuevas agresiones. El miedo a las represalias y a los ataques puede impedir que las víctimas informen de las situaciones de violencia, o pone a las que denuncian esos actos en condiciones de seguridad precarias, lo que las hace más vulnerables a las agresiones (11). Además, la violencia sexual como urgencia médica debe ser atendida con la mayor prontitud, con el fin de evitar consecuencias graves para la salud física y mental que se pueden derivar de este acto. Una atención oportuna y de calidad dentro de las 72 horas posteriores a la agresión reduciría el riesgo de contraer enfermedades, infecciones de transmisión sexual y embarazos no deseados.

Según los resultados de esta investigación, los conocimientos, actitudes y prácticas de los profesionales de la salud encuestados en el departamento de Caquetá ponen en evidencia que debe trabajarse el tema de la IVE en aras de promover el acceso de las mujeres a este servicio. Aunque el $74 \%$ de los profesionales manifestó que habían recibido capacitación en el abordaje de violencia sexual, desconocen que la denuncia es el único requisito de ley para acceder a la IVE, siempre y cuando haya garantías para entablarla sin que represente un peligro para la vida de la mujer. Esto se podría convertir en una barrera para acceder al servicio o hacerlo de manera oportuna. Este resultado difiere con el estudio de Goldman y colegas realizado en Brasil (12), en el cual el $70 \%$ de los participantes no había recibido entrenamiento en el tema. Sin embargo, comparado con el mismo estudio, el $86 \%$ de los profesionales conocía de la aspiración manual endouterina (AMEU) como método para practicar la IVE, mientras en este estudio el $50 \%$ de los participantes desconoce este método. Si bien, tanto la AMEU como el método de dilatación y curetaje se encuentran dentro del plan obligatorio de salud colombiano, según lo cita Prada "se requeriría establecer mecanismos para asegurar que se cumpla con las directrices médicas para la interrupción voluntaria del embarazo (IVE) emitidas por el Ministerio de Salud y Protección Social” (13).

Los profesionales de este estudio opinan que si existe violación se debe recibir atención médica y psicológica. Cuando se les preguntó sobre lo que debe hacer una mujer embarazada a causa de una violación refirieron que lo que ella considere correcto, lo cual sugiere que empieza a tenerse en cuenta la autonomía de la paciente como primordial en el proceso. Igualmente, al preguntar por lo que debe pasar cuando una mujer solicita la IVE, no se evidenció censura alguna ya que mayoritariamente coincidieron en que debe recibir orientación, aun cuando se planteaban respuestas como que debe ir a la cárcel o ser operada para no tener más hijos. Este hallazgo parece sugerir que, al margen de los prejuicios personales, la actuación del profesional en estos casos se soporta primordialmente en principios de la ética profesional y de la bioética.

Para el 58\% de los profesionales encuestados la permisividad de la ley en cuanto al aborto sería directamente proporcional a la práctica de la IVE, mientras que en un estudio en Perú sobre conocimientos actitudes y prácticas de médicos ginecoobstetras con relación al aborto (14), un $29 \%$ cree que este aumentaría si la ley fuera más permisiva. Para el caso de esta investigación se 
puede inferir que los profesionales consideran que el aborto se realiza sólo cuando es legalizada esta práctica, mientras que en el estudio del Perú consideran que la legalidad de la práctica no interviene en el número de solicitudes del procedimiento.

La opinión de los encuestados sobre cuándo debería ser legal la práctica del aborto se centra en casos como violación, cuando corre peligro la vida de la mujer o el feto presenta malformación, con un $23 \%$ de aceptación para cada caso. Esto concuerda con un estudio cualitativo realizado en México (15) con personal de salud sobre la experiencia en el programa de interrupción legal del embarazo, quienes manifestaban que apoyaban la práctica del aborto en casos de violación, malformaciones congénitas o cuando corría peligro la vida o salud de la mujer; esta opinión se podría deber a que estas situaciones son consideradas de mayor afectación sobre la salud física y mental de las mujeres. Sin embargo, difiere con la opinión de los profesionales que participaron en el estudio de Rivarola Espinosa en Paraguay (16) en el cual el 80 \% manifestó que no está de acuerdo en que debería practicarse el aborto si la mujer padece de una enfermedad, mientras que el $78 \%$ considera injustificado el aborto eugenésico y el $77 \%$ no ve viable el aborto cuando el embarazo ha sido resultado de una violación; las diferencias anteriores pudieran deberse a las características culturales de las poblaciones estudiadas acerca de la valoración de la vida humana ya sea desde el momento de la concepción o desde el nacimiento.

Con respecto a la práctica en la atención a menores de 14 años, los profesionales priorizan la atención por parte del Instituto Colombiano de Bienestar Familiar, lo cual supone una dilación en la atención y dejar pasar un tiempo importante para la recolección de muestras forenses. En situaciones de víctimas de violación en general que soliciten la atención en menos de 72 horas después de ocurrida, los encuestados entienden claramente que primero se deberá brindar la atención y luego orientar para la denuncia.

Si bien el $15 \%$ de los participantes se ha declarado como objetor de conciencia, el $47 \%$ de ellos manifestó que no practicaría una IVE, sino que preferiría remitirla. El derecho al acceso a este procedimiento entra en un campo de negociación (17) entre la decisión de la mujer y las barreras que le pueda ocasionar el derecho médico a objetar, al no encontrar una pronta respuesta a dicha solicitud.

\section{CONCLUSIONES}

Los profesionales encuestados tienen información relacionada con la atención a víctimas de violencia sexual. Aun así, hay algunas dificultades en el reconocimiento de los métodos para la IVE y de los requisitos para prestar este servicio. A esto se suma la particularidad de que el departamento de Caquetá es zona de conflicto armado, lo cual hace que estos requisitos sean valorados dependiendo de la causal por la cual se solicita. Además, se logró evidenciar que los profesionales prefieren remitir los casos para la realización de la IVE.

Resulta necesario empoderar a las mujeres sobre derechos sexuales y reproductivos, así como divulgar la norma que legaliza la práctica del aborto en sus tres causales. Esto permitiría disminuir las barreras por parte de los profesionales, al encontrar una comunidad informada. Asimismo, es recomendable que las instituciones prestadoras de servicios de salud realicen un continuo seguimiento al cumplimiento de las normas por parte de su personal, lo cual contribuye a garantizar los derechos de las pacientes, además de incrementar la calidad de la atención ofrecida.

Con relación a la ampliación del conocimiento obtenido en esta investigación, se recomienda hacer un análisis de las características sociodemográficas para relacionar los conocimientos, actitudes y prácticas con variables como sexo, edad o experiencia laboral, entre otras.

\section{REFERENCIAS BIBLIOGRÁFICAS}

1. OMS. Aborto sin riesgos: guía técnica y de políticas para sistemas de salud. En: Salud OMdl. Aborto sin riesgos: guía técnica y de políticas para sistemas de salud. Ginebra; 2012. p. 124.

2. Organización Mundial de la Salud. Prevención del aborto peligroso. Nota descriptiva [Internet]. Ginebra, Organización Mundial de la Salud; 2016 [consultado 19 de diciembre de 2016]. Disponible en: http://www.who. int/mediacentre/factsheets/fs388/es/ 
3. Kane G, Galli B, Skuster P. Cuando el aborto es un crimen: la amenaza para mujeres vulnerables en América Latina. Resumen ejecutivo. Chapel Hill, Carolina del Norte: IPAS; 2013.

4. Lindsey-Curtet C, Holst-Roness FT, Anderson L. Responder a las necesidades de las mujeres afectadas por conflictos armados. Guía Práctica. [Internet]. Ginebra, Comité Internacional de la Cruz Roja; 2006 [consultado 19 de diciembre de 2016]. Disponible en: https://www.icrc.org/spa/assets/files/other/icrc_003 _0840.pdf

5. Ministerio de la Protección Social, Universidad Nacional Colombia. Implicaciones éticas, jurídicas y médicas de la Sentencia C-355 de la Corte Constitucional: Un avance para el ejercicio de los derechos humanos, sexuales y reproductivos de las colombianas. Guía técnica. Bogotá: Editorial Kimpres; 2007. p. 96.

6. Araújo J, Vargas C. Corte Constitucional. Sentencia C-355. Colombia; 2006.

7. Martínez A. Toda Colombia [Internet]. Bogotá: 2015 [consultado 19 de diciembre de 2016]. Disponible en: http://www.todacolombia.com/departamentos-decolombia/caqueta.html

8. Procuraduría General de la Nación, Procuraduría Delegada para la Defensa de los Derechos de la Infancia, la Adolescencia y la Familia. Informe de vigilancia a la Sentencia C- 355 de 2006. Bogotá; 2010.

9. La Mesa por la Vida y la Salud de las Mujeres. El acceso al aborto seguro y su impacto en la salud de las mujeres en Colombia [Internet]. Bogotá: 2015 [consultado 10 de enero de 2017] Disponible en: http://www.clacaidigital. info:8080/xmlui/handle/123456789/154
10. República de Colombia, Ministerio de Salud. Resolución N.º 8430 del 4 de octubre de 1993 [Internet]. 1993 [consultado 7 de enero de 2017]. Disponible en: https://www.minsalud. gov.co/Normatividad_Nuevo/RESOLUCION\%208430\%20 DE\%201993.pdf

11. Villellas, M. La violencia sexual como arma de guerra [Internet]. España: Escola de Cultura de Pau; 2010 [consultado 29 de noviembre de 2016]. Disponible en: http://escolapau.uab.es/img/qcp/violencia_sexual_ guerra.pdf

12. Goldman L, García S, Díaz J, Yam E. Brazilian obstetriciangynecologists and abortion: a survey of knowledge, opinions and practices. Reproductive Health. 2005 nov:;2(10):21.

13. Prada E, Singh S, Remez L, Villarrreal L. Embarazo no deseado y aborto inducido en Colombia: causas y consecuencias. Nueva York: Guttmacher Institute; 2011. p. 21.

14. Távara Orozco L, Sacsa Delgado D. Conocimientos, actitudes y prácticas de médicos ginecoobstetras peruanos en relación al aborto. Lima: Promsex; 2008. p. 52.

15. Díaz Olavarrieta C, Cravioto VM, Villalobos A, DeebSossa N, García L, García S. El programa de interrupción legal del embarazo en la ciudad de México: experiencias del personal de salud. Revista Panamericana de Salud Pública. 2012dic.; 32:6.

16. Rivarola J. Conocimientos y actitudes de los profesionales de la salud sobre el aborto provocado en Paraguay. Anales de la Facultad de las Ciencias Médicas. 2010 jun.; 43(1):12.

17. Casas L, Dides C. Objeción de conciencia y salud reproductiva en Chile: dos casos paradigmáticos. Acta Bioethica. 2007; 13(2): 8. 